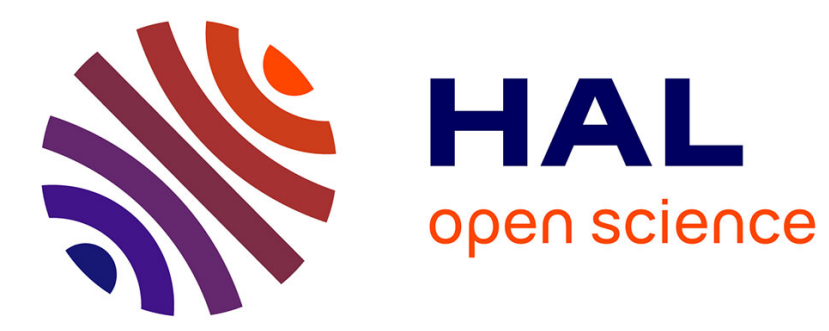

\title{
Depletion forces induce visco-elasto-capillary thinning of non-Brownian suspensions
}

\author{
R. Harich, A Deblais, A Colin, H. Kellay
}

\section{To cite this version:}

R. Harich, A Deblais, A Colin, H. Kellay. Depletion forces induce visco-elasto-capillary thinning of non-Brownian suspensions. EPL - Europhysics Letters, 2016, 114 (5), pp.58006 (1-6). 10.1209/02955075/114/58006 . hal-01392221

\section{HAL Id: hal-01392221 \\ https://hal.science/hal-01392221}

Submitted on 4 Nov 2016

HAL is a multi-disciplinary open access archive for the deposit and dissemination of scientific research documents, whether they are published or not. The documents may come from teaching and research institutions in France or abroad, or from public or private research centers.
L'archive ouverte pluridisciplinaire HAL, est destinée au dépôt et à la diffusion de documents scientifiques de niveau recherche, publiés ou non, émanant des établissements d'enseignement et de recherche français ou étrangers, des laboratoires publics ou privés.

\section{다(1)(2)}

Distributed under a Creative Commons Attribution - ShareAlikel 4.0 International 


\title{
Depletion forces induce visco-elasto-capillary thinning of non-Brownian suspensions
}

\author{
R. Harich ${ }^{1}$, A. Deblais ${ }^{1}$, A. Colin $^{2}$ and H. Kellay ${ }^{1}$ \\ 1 Université de Bordeaux, Laboratoire Ondes et Matière d'Aquitaine, (UMR 5798 CNRS) - 351 cours de la Libération, \\ 33405 Talence France \\ 2 ESPCI, CNRS, SIMM UMR 7615 - 11 rue Vauquelin, 75005 Paris, France
}

PACS 82.70.Kj - Emulsions and suspensions

PACS 83.80.Rs - Polymer solutions

PACS 83.50.Jf - Extensional flow and combined shear and extension

\begin{abstract}
Droplet pinch-off, which occurs when a drop of liquid detaches from a capillary, can be strongly modified in the presence of complex fluids such as polymer solutions and suspensions giving rise to long and slender filaments that thin slowly in time. While for polymers, the molecular conformations of the molecules in the filament are responsible for such a behavior, in suspensions the mechanisms at play remain to be deciphered. Here we show, experimentally, that while liquid bridges of non-Brownian suspensions of moderate concentrations have a thinning behavior very close to that of the solvent, the addition of short-chain polymers inducing depletion attractions between the particles in the suspension changes the thinning dynamics and gives rise to exponential thinning in time. The characteristic time of this dynamics increases with polymer concentration and therefore the intensity of the depletion forces at play. The tunability of this dynamics may be important for injket and 3D printing applications where short rupture times are sought for or in other situations where drop formation has to be minimized or inhibited.
\end{abstract}

Complex fluids exhibit an array of properties that set them apart from Newtonian fluids like water $[1,2]$. A major difference between these types of liquids is the presence of mesoscopic constituents, such as polymers, surfactant aggregates, colloidal or non-Brownian particles which render the interaction of the complex fluid with any perturbation complicated due to the multiplicity of length and time scales that may come into play $[1,2]$. The understanding of the complicated interactions between the constituents in a complex fluid as well as their interactions with an external perturbation such as a flow field is essential for the taming of such fluids which are relevant to a variety of applications. Particle suspensions are an important class of complex fluids, they are familiar to us in our daily lives and extremely important for industrial applications in the food industry, in paint production and for building materials. They are mixtures of soft or solid particles in a liquid with the particle volume fraction playing an important role in determining their behavior: at low volume fraction they behave like a Newtonian fluid with a constant viscosity but at high enough volume fractions, these systems have other rheological responses and notably shear thickening [3-5]. In general model suspensions are constituted of non-Brownian particles with no particular interactions apart from hard sphere repulsion. Real suspensions on the other hand are made of particles and other entities and interactions between the constituents may be important.

In a model suspension of particles the interactions can be tuned by different methods such as the addition of polymers, the addition of salts, or by designing particles with embedded properties for example. These additives or modifications give rise to (or inhibit) different particle-particle interactions such as depletion forces, hydrophilic/hydrophobic interactions, electrostatic interactions, or magnetic interactions among others. Understanding and controlling the role of interparticle interactions in the behavior of suspensions is essential in numerous areas of technology, ranging from inkjet printing to the behavior of concentrated bacterial solutions for biotechnology [6,7]. Different studies have focused on the role of interactions in setting the shear rheology of suspensions and important results on the role of interactions in setting the yield stress or the shear thickening behavior of 
these suspensions have been obtained [8]. Much less work concerns their extensional behavior and its modification by particle interactions.

A powerful way to study the extensional behavior of complex fluids is the thinning and the ensuing rupture of liquid columns [2,9-22]. The rupture of Newtonian liquid cylinders can be modeled using self-similar solutions for the neck profile and power laws for the thinning of the neck [23]. Some complex fluids, however, exhibit an inhibition of such singular dynamics which is replaced, in a sudden fashion, by single time dynamics represented by exponential thinning of the neck [15-18]. Different classes of complex fluids have shown such a phenomenology: polymer solutions, corn starch solutions [24], and micellar phases of surfactant [25]. Even more recently, concentrated colloidal suspensions and nonBrownian suspensions of particles have been shown to exhibit such a behavior $[24,26]$. This exponential thinning has become a powerful tool to characterize complex fluids $[2,16,18-20,27]$. Despite the generality of such a thinning law, known as the visco-elasto-capillary regime, the exact reasons behind its existence or the tailoring of fluid properties to obtain such a law has been lacking [2]. Only few experiments make a link between such a behavior and the interaction between the flow and the dynamics of the constituents of the complex fluid. Such work has been carried out for polymer solutions [22] but for other classes of complex fluids such as suspensions much less is known. For example in two recent papers, the exponential behavior signaling the presence of extensional stresses has been observed in corn starch suspensions as well as concentrated non-Brownian and Brownian suspensions [24,26]. A link has been made with the normal stresses in the medium but the exact role of these stresses in the appearance of this thinning law is still unclear.

Here we show that the introduction of attractive forces between otherwise non-interacting particles can induce this exponential thinning behavior. In order to do so we use suspensions of non-Brownian particles suspended in short-chain polymer solutions at different concentrations. Our main observation is that while the solvent (water), the polymer solution, or the suspension in water at volume fractions up to $40 \%$ all behave as inviscid liquids for the pinch-off behavior, the suspension with addition of polymers shows a much slower thinning dynamics which is well approximated by visco-elasto-capillary pinch-off. This transition occurs for relatively low polymer concentrations for which the polymer solution shows a power law thinning behavior. The characteristic time of the observed exponential thinning increases with increasing polymer concentration and increasing particle volume fraction. The change in polymer concentration allows to tune the depletion potential between the particles and the characteristic time is found to increase linearly with the depth of this potential. We suggest that the formation of aggregates and their eventual breakup by the extensional flow in the liquid bridge is at the origin of this behavior.



Fig. 1: (Colour online) Pictures showing droplet breakup for (a) water at $2.47,0.82,0.082$ and $0 \mathrm{~ms}$ before rupture, (b) polymer solution at $4.77,1.48,0.66$ and $0.083 \mathrm{~ms}$ before rupture, (c) particle suspension at 2.16, 1.08, 0.54 and $0.027 \mathrm{~ms}$ before rupture, (d) polymer-particle suspension at $16.75,8.42$, 3.42 and $1.75 \mathrm{~ms}$ before rupture. The width of the images is $1.65 \mathrm{~mm}$ except for (b) for which it is $2.25 \mathrm{~mm}$. Thinning curves $D_{\min }^{3 / 2}$ as a function of $t_{c}-t$ for particle suspensions at different volume fractions (e) and polymer solutions at different concentrations (f). The dashed lines show the expected inertial-capillary thinning law before neck breakup with a surface tension of $70 \mathrm{mN} / \mathrm{m}$.

Our solutions were prepared using PMMA particles with a diameter of $6 \mu \mathrm{m}$, a short-chain polymer PEG of molecular weight 35000 in water. Glycerol (at $20 \%$ by weight) was added to the water to minimize the sedimentation of particles. The PMMA particles, obtained as a powder, were washed several times and centrifuged in water to obtain a concentrated paste. The polymer was used as received and dissolved in water at different concentrations. Solutions of different concentrations of polymer in water and for different particle volume fractions were then prepared. The solutions were made to drip from a capillary of $4 \mathrm{~mm}$ diameter connected to a syringe. A syringe pump was used to inject the solution (at rates of $0.5 \mathrm{ml} / \mathrm{h}$ ) and induce droplet detachment. Images were recorded using a fast camera (Phantom V641) working at rates up to 50000 frames/s and equipped with different lenses allowing to reach a spatial resolution near $10 \mu \mathrm{m}$ per pixel.

Our main observation is reported in the images of fig. 1. Here a sequence of images at different times before rupture 
is displayed for the pure solvent (water), for the polymer solution, and for the suspension. All three cases show similar pinch-off behavior and dynamics. The behavior obtained is that of rupture controlled by inertia and capillarity at the late instants before neck breakup. The minimum neck diameter in this case varies in time $t$ as $D_{\text {min }}=0.7(\gamma / \rho)^{1 / 3}\left(t_{c}-t\right)^{2 / 3}$, where $\gamma$ is the surface tension, $\rho$ is the density and $t_{c}$ is the breakup time. The data for the three samples (water, suspensions of different volume fractions (e) and different-concentration polymer solutions (f)) agrees with this expression as shown in fig. 1(f) albeit for the constant 0.7; the dashed lines use 0.8 which seems to give a better agreement for a surface tension of $70 \mathrm{mN} / \mathrm{m}$. Variations in slope between the different samples may be due to small variations in this surface tension. The polymer solutions and the suspensions behave as inviscid fluids as found previously [26] for volume fractions less than $45 \%$. When the polymer is added to the suspension, the behavior changes. The rupture time increases drastically and the neck elongates to form a long filament reminiscent of visco-elasto-capillary thinning often observed with polymer solutions [2]. While the neck shapes at breakup are similar for the three control samples as expected for the inertial capillary regime (fig. 1(e)), the suspension with added polymer has a neck shape that is drastically different, symmetric, and cylindrical. This large change in behavior is obtained with other combinations of polymer concentration and particle volume fraction.

To quantify this behavior, we plot, in fig. 2(a), the thinning dynamics of the different solutions. This dynamics is fast for the first three solutions (water, polymer solution, and the suspension), follows the $2 / 3$ power law characteristic of inertial capillary thinning, but becomes slower and slower as the concentration of polymer in the suspension increases. This slowing-down is much more important compared to the polymer solution behavior as illustrated in this figure where a polymer solution ruptures much faster than a suspension with much less polymer. Figure 2(b) shows the slowing-down caused by the increase in the volume fraction of the particles while the polymer concentration varies over a small range. Even if the polymer slows down the dynamics, the slowing-down caused by the presence of the particles is much more important and increases in importance (with respect to the suspension without added polymer or to the polymer solution itself) as the suspension volume fraction increases. Note here that the last instants before breakup seem to follow an inertial capillary thinning dynamics as observed before for suspensions. For these last instants, the decrease of the suspension volume fraction is generally observed giving rise to dynamics dominated by the solvent properties $[11,28,29]$. Figure 2(c) shows the thinning dynamics of 2 suspensions at a volume fraction of $35 \%$ with 60 and $120 \mathrm{mg} / \mathrm{mL}$ of added polymer. Different thinning laws are used to model the full thinning dynamics; the latest instants are fit to a power law dynamics with an exponent
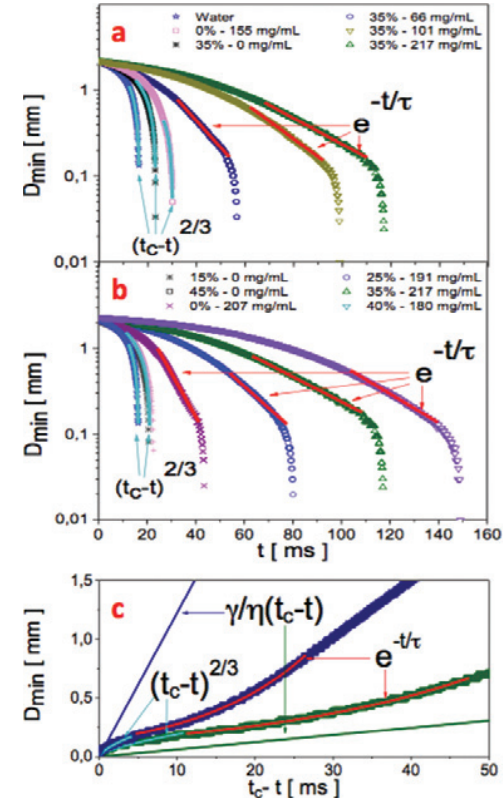

Fig. 2: (Colour online) Thinning curves $D_{\min }$ as a function of $t$ for the different solutions used. (a) Effect of polymer concentration on the breakup of a suspension at $35 \%$ volume fraction. Water, the polymer solution, and the suspension without addition of polymers are also displayed. (b) Effect of polymer on suspensions with different volume fractions. Water (same symbol as in (a)), suspensions without polymer as well as the polymer solution are also shown. The red lines indicate the thinning region where an exponential law is used to approximate the thinning behavior. The final instants show fast thinning reminiscent of inertial capillary breakup. (c) Thinning curves $D_{\min }$ as a function of $t_{c}-t$, the light blue lines at the lastest instants before breakup are fits with power law of $2 / 3$ and the red lines at intermediate times are exponential fits, the green and blue straight lines show the expected visco-capillary breakup with a linear thinning law $0.14 \frac{\gamma}{\eta}\left(t_{c}-t\right)$. Here we have used a surface tension $\gamma=72 \mathrm{mN} / \mathrm{m}$ and solution viscosities $\eta$ of 0.1 (for the $60 \mathrm{mg} / \mathrm{mL}$ solution) and $1.6 \mathrm{~Pa} \cdot \mathrm{s}$ (for the $217 \mathrm{mg} / \mathrm{mL}$ solution).

of $2 / 3$ characteristic of the inertial-capillary regime while an exponential thinning law is used to approximate the dynamics at intermediate times. The straight lines in this figure are the expected visco-capillary breakup dynamics with a linear thinning law $\mathrm{D}_{\min }=0.14 \frac{\gamma}{\eta}\left(t_{c}-t\right)$, with the viscosity $\eta$ determined using rheology measurements to be shown below. Note that the solutions used have dynamics which is difficult to reconcile with the viscocapillary thinning expected for the Newtonian suspensions studied here. The indicated exponential thinning works better.

Two important features characterize the thinning curves in the presence of particles and polymers shown in fig. 2(a) and (b). While at the late times before rupture, the dynamics seems similar and fast for all volume fractions, the intermediate time region shows a roughly exponential behavior. The dynamics for this part can be reasonably approximated by $D_{\min }=A \exp (-t / \tau)$. 


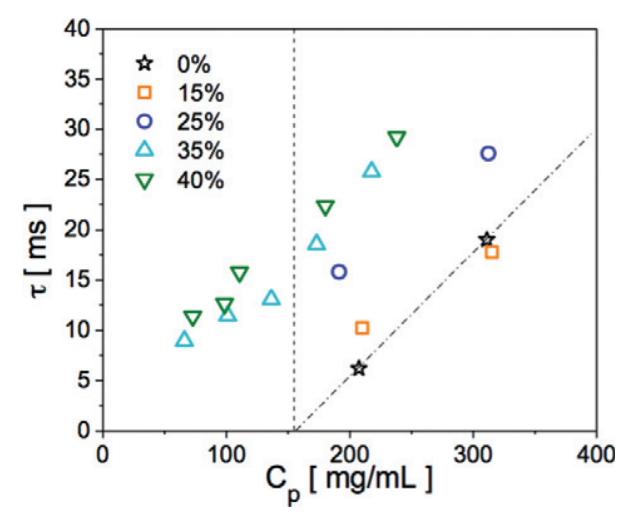

Fig. 3: (Colour online) Decay time of the exponential thinning regime vs. polymer concentration and for different suspension volume fractions. Also reported in this graph, the decay times of the polymer solution without added particles, the dot-dashed line. The vertical dashed line indicates the polymer concentration below which no exponential thinning is observed for the polymer solution without added particles.

This regime appears linear in the semi-log representation of fig. 2. The characteristic time $\tau$ of this exponential regime increases with polymer concentration and with particle volume fraction as seen in fig. 2. The variation of this characteristic time is displayed in fig. 3 vs. polymer concentration and for different volume fractions of particles. Note that for each volume fraction this time increases roughly linearly with polymer concentration. Also, and for the smallest volume fraction (15\%), the characteristic time is relatively close to that measured in the bare polymer solution for high enough concentrations; the exponential regime is not observed for polymer concentrations below a certain value (vertical dashed line) for the low suspension volume fractions or for polymer solutions without added particles. Otherwise and for higher volume fractions $(>15 \%)$, the exponential regime is present even for polymer concentrations not exhibiting the exponential regime. The appearance of this exponential regime seems to occur above a threshold polymer concentration which increases as the volume fraction decreases. For the $15 \%$ suspensions, this transition occurs very close to the concentration for which the bare polymer solution also shows a transition to an exponential regime. This indicates that the effect at $15 \%$ is difficult to attribute to the mixture instead of the polymer alone; even the characteristic times seem to be very close. For the higher volume fractions the effect is very clear and robust and even if the polymer solution shows an exponential regime for high enough polymer concentrations, the characteristic time of the polymer laden suspension is much larger.

The question then is why the mixture (polymer and added particles) gives rise to such a slowing-down of its thinning behavior which is characteristic of viscoelastic fluids such as polymer solutions? Observations of similar exponential thinning in colloidal as well as non-Brownian suspensions have been made but at

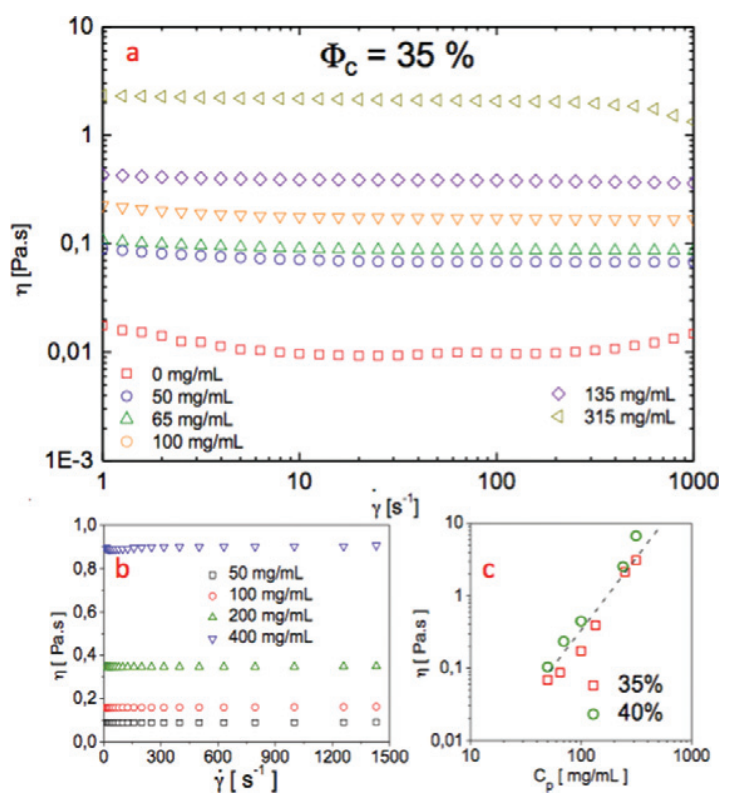

Fig. 4: (Colour online) Rheological measurements: (a) Shear viscosity vs. shear rate for $35 \%$ suspensions with different polymer concentrations. (b) Shear viscosity vs. shear rate for polymer solutions. (c) Suspension viscosity vs. polymer concentration for 35 and $40 \%$ volume fractions. The dashed line indicates a quadratic increase of the viscosity vs. polymer concentration.

concentrations sufficiently high that normal stresses are important. The emergence of this behavior, at least for non-Brownian suspensions, has been shown to be directly related to the appearance of positive normal stresses in the system $[24,26]$. The characteristic time of the exponential dynamics in such systems has been found to correlate very well with the inverse shear rate, $\dot{\gamma}_{N}$, for the onset of positive normal stresses $\left(3 / \dot{\gamma}_{N}=\tau\right)$ pointing to their role in setting the characteristic time of viscoelastic pinch-off $[24,26]$. A priori, and for the volume fractions used here $(\leq 40 \%)$, the viscosity of the solutions is constant and independent of shear rate and the onset of positive normal stresses does not occur (or if it occurs it does so at much higher shear rates) according to our rheological measurements shown in fig. 4. Since at onset of positive normal stresses, concentrated solutions usually show a shear thickening behavior, our solutions which remain Newtonian at shear rates higher than $3 / \tau$ as shown in figs. 3 and 4(a) do not obey this simple criterion. The viscosity of the polymer laden suspensions is roughly constant for shear rates in the range 1 to $1000 \mathrm{~s}^{-1}$, fig. 4(a), and simply increase as the polymer concentration increases as found in other measurements [30]. The bare polymer solutions (without added particles) are also Newtonian in this range of shear rates, fig. 4(b). Some shear thinning is observed for the polymer laden suspensions but only for high polymer concentrations. Our own measurements of normal stresses in these solutions do not show positive normal stresses and when they are present seem to 




Fig. 5: (Colour online) Pictures of an aggregate of two particles in the thinning neck of a low volume fraction suspension (less than 1\%) in the presence of $200 \mathrm{mg} / \mathrm{mL}$ of polymer, note that the aggregate slowly separates as the thinning of the neck proceeds.

be rather small and negative. Other explanations need to be found for the appearance of the exponential thinning regime.

The main effect of the polymer in such mixtures is to introduce depletion interactions between particles $[31,32]$. As they come close to each other during the flow (they are non-Brownian but in the flowing suspensions relative movements of particles may occur), the particles may stick to each other and eventually form clusters. The strength of the depletion potential in the chosen case (small radius of gyration compared to particle radius) is very large making these aggregates quite strong. The extensional flow in the neck will end up breaking these aggregates. We have confirmed this scenario by using small volume fraction samples (less than 1\%) and observed this behavior by video imaging as shown in the series of photographs in fig. 5 where a cluster of two beads is seen to undergo separation during the thinning of the neck. Experiments using biofibers [33] have also used extensional thinning to break the fibers. Along these lines, we suggest an argument leading to the thinning law observed here. The extensional stress $\eta \dot{\epsilon}$ in the liquid bridge is balanced by the stress needed to break the aggregates. The stress due to the presence of numerous pairs of particles held together by the depletion attraction due to the presence of polymers is proportional to the depletion force $F_{d e p}$ given by $\pi n_{p} k T R R_{g}$ for $\xi=\frac{R_{g}}{R} \ll 1$ (for the 35000 molecular-weight PEG this ratio is $3 \cdot 10^{-3}$ in the dilute regime) [34]. Here $k$ is the
Boltzman constant, $T$ is the temperature, $n_{p}$ is the polymer number density in the free volume, $R$ is the radius of the particle and $R_{g}$ the polymer radius of gyration for low $n_{p}$ or a characterisitic blob size for high $n_{p}$ [34,35]. The extension rate is $\dot{\epsilon}=-\frac{2}{h_{\min }} \frac{\mathrm{d} h_{\min }}{\mathrm{d} t}$ with $h_{\min }=D_{\min } / 2$ and the extensional viscosity $\eta$ of the suspension is assumed to be $3 \eta_{s}$ with $\eta_{s}$ the shear viscosity of the polymer laden suspension as for a Newtonian fluid. From the stress balance we have $-\eta \frac{2}{h_{\min }} \frac{\mathrm{d} h_{\min }}{\mathrm{d} t} \sim \pi n_{p} k T R R_{g} /\left(\alpha 4 \pi R^{2}\right)$. The factor $\alpha 4 \pi R^{2}$ represents the area over which the depletion force acts between two adjacent particles and is taken as a small fraction $\alpha$ of the surface of a particle. This equation has a decaying exponential solution for $h_{\text {min }}$. The decay time of this solution is proportional to $\left(\frac{2 \eta \alpha 4 \pi R^{2}}{\pi n_{p} k T R R_{g}}\right)$. The viscosity of the suspensions at constant volume fraction depends on the polymer concentration $C_{p}$ and therefore on $n_{p}$ in a quadratic fashion (see fig. 4) giving the linear dependence of the decay time on this volume fraction which is born out experimentally. Note that this decay time should increase with the viscosity of the suspension which is also born out experimentally since the decay times increase with the particle volume fraction. Using the measurements of fig. 3 and the rheology measurements, the unkown parameter $\alpha$ turns out to be roughly $10^{-3}$. This gives a depletion force which decays to zero when the separation between two adjacent particles is of roughly one radius of gyration of the polymer molecules which seems reasonable. A further test of this proposal has been carried out by using a smaller-molecular-weight polymer (8000) at a concentration of $200 \mathrm{mg} / \mathrm{ml}$ in a $35 \%$ suspension. By taking into account the variation of $R_{g}$ (or blob size) with $n_{p}$ and the measured $\eta$, we find a ratio of roughly 2.8 for the decay times of the 35000 by the 8000 molecularweight polymers. Experimentally we found a ratio of 2 . The trend is correct and the value is of the same order of magnitude as the calculated ratio but the use of a similar value of $\alpha$ for the two polymer weights is not strictly justified making the estimate just that, an estimate. Note that the extensional stress can be estimated using $\gamma / h_{\min }$, this stress is of order $10^{3} \mathrm{~Pa}$ which is reached in shear rheology measurements only for high suspension volume fractions and high shear rates. This is a probable reason why despite the Newtonian aspect of the solutions, their extensional properties are more sensitive to the presence of aggregates and therefore show dramatic changes in the presence of polymer. While these considerations attempt to link the small scale structures to the macroscopic thinning behavior in a qualitative fashion, a more stringent test where the presence of aggregates is shown directly remains to be done in the concentrated suspensions used here where optical measurements become difficult to carry out. Other scenarios are also possible to explain the emergence of visco-elasto-capillary pinch-off in systems of attractive particles: the polymers and most probably the polymer network may be strongly perturbed in the small spacings between the particles in the presence of relative velocities between these particles and the 
solvent. Nonetheless our simple argument gives the correct qualitative scaling of the thinning time.

In conclusion, we show that the addition of attractive depletion interactions in non-Brownian suspensions gives rise to an elastic regime in the extensional flow of such suspensions which are nonetheless Newtonian under shear. The large difference between the extensional measurements carried out here and the shear measurements of these suspensions brings forth fundamental questions about the nature of the rheological response of particle laden fluids.

We would like to acknowledge the Labex AMADEus, ANR-10-LABX-0042-AMADEUS along with the program of Excellence Initiative Idex Bordeaux, Grant No. ANR10-IDEX-0003-02 for financial support. Z. PAN's help with the rheology measurements is also acknowledged.

\section{REFERENCES}

[1] Larson R. G., The Structure and Rheology of Complex Fluids (Oxford University, New York) 1999.

[2] McKinley G. H., Rheol. Rev. (2005) 1.

[3] Lin N. Y. C. et al., Phys. Rev. Lett., 115 (2015) 228304.

[4] Fall A. et al., Phys. Rev. Lett., 100 (2008) 018301.

[5] Seto R. et al., Phys. Rev. Lett., 100 (2013) 018301.

[6] Kulkarni A. M. et al., Phys. Rev. Lett., 83 (1999) 4554.

[7] Tanaka S. and Ataka M., J. Chem. Phys., 117 (2002) 3504.

[8] Brown E. et al., Nat. Mater., 9 (2010) 220.

[9] Miskin M. Z. and Jaeger H. M., Proc. Natl. Acad. Sci. U.S.A., 109 (2012) 4389.

[10] Furbank R. J. and Morris J. F., Phys. Fluids, 16 (2004) 1777.

[11] Bertrand T. et al., Granular Matter, 14 (2012) 169.

[12] Sмiтh M. I. et al., Nat. Commun., 1 (2010) 114.
[13] Doshi P. et al., J. Non-Newtonian Fluid Mech., 113 (2003) 1; Doshi P. and Basaran O. A., Phys. Fluids, 16 (2004) 585; Suryo R. and Basaran O. A., J. NonNewtonian Fluid Mech., 138 (2006) 134.

[14] Huisman F. M., Friedman S. R. and Taborek P., Soft Matter, 8 (2012) 6767.

[15] Amarouchene Y. et al., Phys. Rev. Lett., 86 (2001) 3558.

[16] Anna S. L. and McKinley G., J. Rheol., 45 (2001) 115.

[17] Sattler R., Kityk A. and Wagner C., Phys. Rev. E, 75 (2007) 1.

[18] McKinley G. H. and Sridhar T., Annu. Rev. Fluid Mech., 34 (2002) 375.

[19] Stelter M. et al., J. Rheol., 46 (2002) 507.

[20] Entov V. M. and Hinch E. J., J. Non-Newtonian Fluid Mech., 72 (1997) 31.

[21] Louvet N., Bonn D. and Kellay H., Phys. Rev. Lett., 113 (2014) 218302.

[22] Ingremeau F. and Kellay H., Phys. Rev. X, 3 (2013) 041002.

[23] Eggers J., Rev. Mod. Phys., 69 (1997) 1833; Eggers J. and Villermaux E., Rep. Prog. Phys., 71 (2008) 036601.

[24] Roché M., Kellay H. and Stone H. A., Phys. Rev. Lett., 107 (2011) 134503.

[25] Rothstein J. P., J. Rheol., 47 (2003) 1227.

[26] PAN Z. et al., Phys. Rev. E, 92 (2015) 052203.

[27] Tirtantmadja V. T. and Sridhar T., J. Rheol., 37 (1993) 1081.

[28] Mathues W. et al., Phys. Fluids, 27 (2015) 093301; McIlroy C. and Harlen O. G., Phys. Fluids, 26 (2014) 033101.

[29] Zhао H. et al., Phys. Fluids, 27 (2015) 063303.

[30] Abdul Haleem B. and Nott Prabhu R., J. Rheol., 53 (2009) 383.

[31] Lekkerkerker H. N. W. and Tuinier R., Colloids and the depletion interaction, in Lect. Notes Phys., Vol. 833 (Springer) 2011.

[32] Bergenholtz J., Poon W. C. K. and Fuchs M., Langmuir, 19 (2003) 4493.

[33] Kroes-Nijboer A. et al., Langmuir, 26 (2010) 13097.

[34] Verma R. et al., Phys. Rev. Lett., 81 (1998) 4004.

[35] Li J. et al., Polymer, 80 (2015) 205. 